\title{
The effects of RF impairments in Vehicle-to-Vehicle Communications
}

\author{
Alexandros-Apostolos A. Boulogeorgos ${ }^{1}$, Paschalis C. Sofotasios ${ }^{1,2}$, Sami Muhaidat ${ }^{3,4}$, Mikko Valkama $^{2}$, \\ and George K. Karagiannidis ${ }^{1,3}$ \\ ${ }^{1}$ Department of Electrical and Computer Engineering, Aristotle University of Thessaloniki, 54124 Thessaloniki, Greece \\ e-mail: \{ampoulog; sofotasios; geokarag\} @auth.gr \\ ${ }^{2}$ Department of Electronics and Communications Engineering, Tampere University of Technology, 33101 Tampere, Finland \\ e-mail: \{paschalis.sofotasios; mikko.e.valkama\} @tut.fi \\ ${ }^{3}$ Department of Electrical and Computer Engineering, Khalifa University, PO Box 127788, Abu Dhabi, UAE \\ ${ }^{4}$ Department of Electronic Engineering, University of Surrey, GU2 7XH, Guildford, United Kingdom \\ e-mail: muhaidat@ieee.org
}

\begin{abstract}
Radio frequency (RF) front-ends constitute a fundamental part of both conventional and emerging wireless communication systems. However, in spite of their importance they are often assumed ideal, although they are practically subject to certain detrimental impairments, such as amplifier nonlinearities, phase noise and in phase and quadrature (I/Q) imbalance (IQI). The present work is devoted to the quantification and evaluation of the RF IQI effects in the context of realistic wireless vehicle-tovehicle (V2V) communications over double-Nakagami $-m$ fading channels. Novel closed form expressions are derived for the corresponding outage probability for the case of ideal transmitter (TX) and receiver (RX), ideal TX and I/Q imbalanced $R X, I / Q$ imbalanced TX and ideal RX, and joint I/Q imbalanced TX/RX. The offered analytic results have a relatively convenient algebraic representation and their validity is extensively justified through comparisons with respective results from computer simulations. Based on these, it is shown that cascaded fading results to considerable degradations in the system performance and that assuming ideal RF front-ends at the TX and RX induces nonnegligible errors in the outage probability evaluation that can exceed $20 \%$ in several $\mathrm{V} 2 \mathrm{~V}$ communication scenarios.
\end{abstract}

\section{INTRODUCTION}

It is well known that vehicle-to-vehicle (V2V) communications constitute a fundamental part of emerging communication systems. This also includes intelligent transportation systems (ITSs), which have been attracting considerable attention due to the large number of applications they can be deployed for. It is recalled that transceivers in $\mathrm{V} 2 \mathrm{~V}$ communication systems experience fading effects that typically differ from conventional terrestrial cellular communication scenarios. This difference arises from the mobility and physical location of the involved TX/RX as well as by the presence of reflectors/scatterers in highways and urban environments. Based on this, the omnidirectional TX and RX antennas in these systems are located at relatively low elevations and thus, the corresponding wireless channel has been shown to exhibit a non-stationary behavior. As a consequence, the performance of corresponding communication systems is subject to non- negligible deteriorations in terms of throughput and outage probability $(\mathrm{OP})$, which becomes particularly problematic in certain communication scenarios including safety applications [1]. This is also the case for the detrimental effects of fading conditions [2]-[13]. To this effect, wireless channels in V2V communications should be also accurately characterized and modeled in order to evaluate the performance of these systems precisely and incorporate the essential techniques that are capable of fulfilling the necessary application requirements that ensure efficient and robust wireless transmission.

The performance of $\mathrm{V} 2 \mathrm{~V}$ communication systems has been investigated in several analyses, see e.g. [14]-[16], and references therein, assuming an ideal radio frequency (RF) frontend. However, the system performance is degraded in practice by non-negligible RF impairments, such as high-power amplifier (HPA) nonlinearity, in-phase and quadrature-phase (I/Q) imbalance (IQI), low-noise amplifier (LNA) nonlinearity, antenna coupling, phase noise (PN) and carrier frequency offset (CFO) [17]. In particular, IQI represents the mismatch between analog components in the I and Q branches, which results from the limited accuracy of analog hardware. In this context, the effects of RF impairments were investigated in [18]-[20], while performance degradation due to IQI was investigated in [17], [21]-[27]. Specifically, the authors in [21], [22] quantified the effect of IQI on the performance of multiple-input multiple-output (MIMO), whereas the authors in [17], [23], and [25] investigated the performance of relaying systems in the presence of IQI. The impact of IQI in cognitive radio systems was also analyzed in [20], [28], where it was shown that in a multi-channel environment IQI increases the false alarm probability significantly compared to the simplistic ideal $\mathrm{RF}$ receiver case.

Nevertheless, to the best of the authors' knowledge no analyses have been reported in the open technical literature on the detrimental effects of IQI in V2V communications over cascaded fading channels. Motivated by this, the present work 
is devoted to the analysis and quantification of IQI effects in the context of wireless transmission over double Nakagami- $m$ fading channels. To this end, novel analytic expressions are derived for the corresponding OP considering the following three scenarios: $i$ ) ideal TX with I/Q imbalanced RX; $i i$ ) I/Q imbalanced TX with ideal RX; iii) joint I/Q imbalanced TX and RX. The derived expressions are validated extensively through comparisons with respective results from computer simulations and are subsequently employed in analyzing the corresponding performance providing useful insights for future design and deployments of $\mathrm{V} 2 \mathrm{~V}$ communication systems.

\section{SYSTEM AND SIGNAL MODEL}

In this Section, we consider a V2V communication system, as presented in Fig. 1, where the TX and RX are equipped with a single antenna. In this context, it is essential to firstly revisit the ideal signal model, which is henceforth referred to as ideal $\mathrm{RF}$ front-end, and the realistic IQI signal models in directconversion transmitter (TX) and receiver (RX) scenarios.

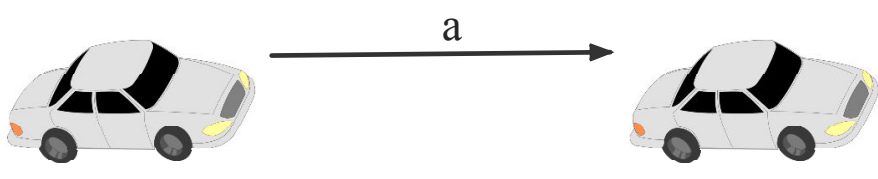

Fig. 1. Indicative V2V Communication Scenario.

\section{A. Ideal RF front-end}

Without loss of generality, the a parameter in Fig. 1 represents the wireless communication link's complex fading coefficient, whose magnitude $h$ is assumed to be the product of statistically independent, but not necessarily identically distributed Nakagami $-m$ random variables ${ }^{1}$, namely [15]

$$
h=\prod_{i=1}^{2} h_{i} .
$$

As already mentioned, due to the increased mobility, the nature of the surrounding environment and the position of the TX and RX antennas in V2V communications, the double Nakagami $-m$ fading distribution has been shown to provide adequate modeling of the corresponding fading effects [15]. Furthermore, we assume a signal, $s$, transmitted over a flat wireless channel, $h$, with additive white Guassian noise, $n$. The received RF signal is passed through various processing stages, also known as the RF front-end of the receiver, and include filtering, amplification, analog I/Q demodulation and sampling. To this effect, the corresponding baseband equivalent received signal is represented as

$$
r_{\text {ideal }}=h s+n
$$

and based on this, the instantaneous signal to noise ratio (SNR) per symbol at the RX input is expressed as

$$
\gamma_{\text {ideal }}=\frac{E_{s}}{N_{0}}|h|^{2}
$$

\footnotetext{
${ }^{1}$ For the special case of $m=1$, the double-Nakagami- $m$ processes reduces to the double Rayleigh processes.
}

where $E_{s}$ denotes the energy per transmitted symbol and $N_{0}$ is the single-sided AWGN power spectral density (PSD). Therefore, the corresponding average SNR is expressed as [29]

$$
\bar{\gamma}=\frac{E_{s}}{N_{0}} \prod_{i=1}^{2} \Omega_{i}
$$

with $\Omega_{i}$ denoting the scaling parameter of the $i^{\text {th }}$ Nakagami $-m$ process.

\section{B. I/Q imbalance Model}

The time-domain baseband representation of the IQI impaired signal is given by [19]

$$
g_{\mathrm{IQI}}=K_{1}^{t / r} g_{\text {id }}+K_{2}^{t / r} g_{\text {id }}^{*}
$$

where $g_{\text {id }}$ denotes the baseband IQI-free signal and $g_{\text {id }}^{*}$ arises due to the involved IQI effects. Furthermore, the IQI coefficients $K_{1}^{t / r}$ and $K_{2}^{t / r}$ are expressed as

$$
K_{1}^{t / r}=\frac{1}{2}\left(1+\epsilon^{t / r} e^{ \pm j \phi^{t / r}}\right)
$$

and

$$
K_{2}^{t / r}=1-\left(K_{1}^{t / r}\right)^{*}
$$

where the positive and negative signs in (6) and the $t / r$ superscripts denote the up and down conversion processes, respectively, whereas the $\epsilon^{t / r}$ and $\phi^{t / r}$ terms account for the TX/RX amplitude and phase mismatch, respectively. Furthermore, the $K_{1}^{t / r}$ and $K_{2}^{t / r}$ coefficients are related to the corresponding image rejection ratio (IRR), which determines the amount of attenuation of the image frequency band, namely

$$
I R R_{t / r}=\frac{\left|K_{1}^{t / r}\right|^{2}}{\left|K_{2}^{t / r}\right|^{2}} .
$$

It is recalled that the second term $K_{2}^{t / r} g_{\mathrm{id}}^{*}$ is caused by the involved imbalances and represents the self-interference effect, while in practical analog RF front-end electronics, the value of IRR is typically in the range of $20 \mathrm{~dB}-40 \mathrm{~dB}$ [30]-[33].

\section{V2V systems impaired by IQI}

This subsection presents the signal model for single-carrier transmission in which the TX and/or the RX is subject to IQI.

1) TX impaired by IQI: In this scenario, it is assumed that TX suffers from IQI while the RF front-end of the RX is ideal. To this effect, it follows from (5) that the baseband equivalent transmitted signal is expressed as

$$
s_{\mathrm{IQI}}=K_{1}^{t} s+K_{2}^{t} s^{*}
$$

whereas the baseband equivalent received signal is given by

$$
r_{\mathrm{IQI}}^{t}=h s_{\mathrm{IQI}}+n=K_{1}^{t} h s+K_{2}^{t} h s^{*}+n .
$$

Furthermore, the instantaneous SINR per symbol at the input of the RX is expressed as

$$
\gamma=\frac{\left|K_{1}^{t}\right|^{2}|h|^{2} E_{s}}{\left|K_{2}^{t}\right|^{2}|h|^{2} E_{s}+N_{0}}
$$


which with the aid of (8) and after basic algebraic manipulations can be re-written as follows:

$$
\gamma=\frac{1}{\frac{1}{I R R_{t}}+\frac{1}{\left|K_{1}^{t}\right|^{2}} \frac{1}{\gamma_{\text {ideal }}}} .
$$

Based on (9) and (12), in the context of direct-conversion transmitter, the IQI effect can be considered as the so-called self-image problem, where the baseband equivalent transmitted signal is interfered by its own complex conjugate [34].

2) RX impaired by IQI: In this scenario, it is assumed that the RX suffers from IQI while the RF front-end is ideal. Based on (5), the baseband equivalent received signal is given by

$$
r=K_{1}^{r} h s+K_{2}^{r} h^{*} s^{*}+K_{1}^{r} n+K_{2}^{r} n^{*}
$$

and the instantaneous SINR per symbol at the RX input is

$$
\gamma=\frac{\left|K_{1}^{r}\right|^{2}|h|^{2} E_{s}}{\left|K_{2}^{r}\right|^{2}|h|^{2} E_{s}+\left(\left|K_{1}^{r}\right|^{2}+\left|K_{2}^{r}\right|^{2}\right) N_{0}}
$$

which with the aid of (8) and after basic algebraic manipulations can be equivalently expressed as follows:

$$
\gamma=\frac{1}{\frac{1}{I R R_{r}}+\left(1+\frac{1}{I R R_{r}}\right) \frac{1}{\gamma_{\text {ideal }}} .}
$$

3) Joint $T X / R X$ impaired by IQI: In this scenario, it is assumed that both TX and RX suffer from IQI. This is a more realistic scenario and based on (5), it follows that the baseband equivalent received signal is given by

$$
r=\left(\xi_{11} h+\xi_{22} h^{*}\right) s+\left(\xi_{12} h+\xi_{21} h^{*}\right) s^{*}+K_{1}^{r} n+K_{2}^{r} n^{*}
$$

where $\xi_{11}=K_{1}^{r} K_{1}^{t}, \xi_{22}=K_{2}^{r}\left(K_{2}^{t}\right)^{*}, \xi_{12}=K_{1}^{r} K_{2}^{t}$ and $\xi_{21}=K_{2}^{r}\left(K_{1}^{t}\right)^{*}$. To this effect, the instantaneous SINR per symbol at the input of the RX is expressed as

$$
\gamma=\frac{|Z|^{2} E_{s}}{|W|^{2} E_{s}+\left(\left|K_{1}^{r}\right|^{2}+\left|K_{2}^{r}\right|^{2}\right) N_{0}}
$$

with $|Z|^{2}=\left|\xi_{11} h+\xi_{22} h^{*}\right|^{2}$ and $|W|^{2}=\left|\xi_{12} h+\xi_{21} h^{*}\right|^{2}$ i.e.

$$
|W|^{2}=\left|\xi_{12}\right|^{2}|h|^{2}+\left|\xi_{21}\right|^{2}|h|^{2}+2 \Re\left\{\xi_{12} \xi_{21} h^{2}\right\}
$$

whereas $\left|\xi_{22}\right|^{2} /\left|\xi_{11}\right|^{2}=1 / I R R_{r} I R R_{t}$ which for practical IQI levels lies in the range of $[-43,-28 \mathrm{~dB}]$. As a result, it readily follows that $|Z|^{2} \approx\left|\xi_{11}\right|^{2}|h|^{2}$ and given that

$$
2 \Re\left\{\xi_{12} \xi_{21} h^{2}\right\}<<\left|\xi_{12}\right|^{2}|h|^{2}+\left|\xi_{21}\right|^{2}|h|^{2}
$$

equation (18) can be expressed as $|W|^{2} \approx\left|\xi_{12}\right|^{2}|h|^{2}+$ $\left|\xi_{21}\right|^{2}|h|^{2}$. Based on the above eq. (17) can be re-written as

$$
\gamma \approx \frac{\left|\xi_{11}\right|^{2}}{\left|\xi_{12}\right|^{2}+\left|\xi_{21}\right|^{2}+\left(\left|K_{1}^{r}\right|^{2}+\left|K_{2}^{r}\right|^{2}\right) \frac{1}{\gamma_{\text {idal }}}} .
$$

which is particularly accurate as the involved relative error does not practically exceed $1 \%$.

\section{OUtAge PRobability in V2V Communications}

It is recalled that the OP can be defined as the probability that the symbol error rate is greater than a certain quality of service level and is computed as the probability that the instantaneous SNR or SINR falls bellow the corresponding predetermined threshold [35]. In what follows, we derive a novel analytical framework for the OP over double Nakagami-m fading channels subject to the aforementioned IQI scenarios in V2V communication systems. The offered analytic expressions are validated through extensive comparisons with respective results from computer simulations.

\section{A. Ideal RF front-end}

It is recalled that the envelope PDF of double Nakagami- $m$ channels i.e. $N=2$, is given in [29, Eq. (6)]. Based on this and with the aid of [36, eq. (2.3)], it immediately follows that

$$
p_{\gamma}(\gamma)=\mathcal{A} \gamma^{\frac{m_{1}+m_{2}}{2}-1} K_{m_{1}-m_{2}}(\mathcal{B} \sqrt{\gamma})
$$

where $K_{n}(\cdot)$ denotes the modified Bessel function of the second kind whereas $\mathcal{A}=2 /\left(\bar{\gamma}^{\frac{m_{1}+m_{2}}{2}} \prod_{i=1}^{2} \Gamma\left(m_{i}\right) m_{i}^{-\frac{m_{1}+m_{2}}{2}}\right)$ and $\mathcal{B}=\prod_{i=1}^{2} m_{i} / \sqrt{\bar{\gamma}}$. Therefore, the corresponding CDF is

$$
F_{\gamma}(x)=\mathcal{A} \int_{0}^{\gamma} x^{\frac{m_{1}+m_{2}}{2}-1} K_{m_{1}-m_{2}}(\mathcal{B} \sqrt{x}) \mathrm{d} x
$$

which can be expressed in closed-form using [37, eq. (1.12.2)]

$$
\begin{aligned}
F_{\gamma}(\gamma)= & \frac{\mathcal{A} \gamma^{m_{1}} \Gamma\left(m_{2}-m_{1}\right)}{m_{1} 2^{m_{1}-m_{2} \mathcal{B}^{m_{2}-m_{1}}}} \\
& \quad \times{ }_{1} F_{2}\left(m_{1} ; m_{1}+1, m_{1}-m_{2}+1 ; \frac{\mathcal{B}^{2} \gamma}{4}\right) \\
+ & \frac{\mathcal{A} \gamma^{m_{2}} \Gamma\left(m_{1}-m_{2}\right)}{m_{2} 2^{1-m_{1}+m_{2}} \mathcal{B}^{m_{2}-m_{1}}} \\
& \times{ }_{1} F_{2}\left(m_{2} ; m_{2}+1, m_{2}-m_{1}+1 ; \frac{\mathcal{B}^{2} \gamma}{4}\right)
\end{aligned}
$$

where ${ }_{p} F_{q}(\cdot)$ denotes the generalized hypergeometric function [37]. In the same context, for the special case that $m_{1}-m_{2} \pm$ $\frac{1}{2} \in \mathbb{N}$, the $K_{n}(\cdot)$ function in (21) can be expressed according to [38, eq. (8.468)]. Based on this and by performing the necessary variable transformation, the SNR PDF of the double Nakagami $-m$ fading model can be alternatively expressed as

$p_{\gamma}(\gamma)=\mathcal{A} \sqrt{\pi} \sum_{l=0}^{m_{1}-m_{2}-\frac{1}{2}} \frac{\left(m_{1}-m_{2}+l-\frac{1}{2}\right) ! \gamma^{\frac{m_{1}+m_{2}-l}{2}}-\frac{5}{4}}{l !\left(m_{1}-m_{2}-l-\frac{1}{2}\right) !(2 \mathcal{B})^{l+\frac{1}{2}} e^{\mathcal{B} \sqrt{\gamma}}}$

whereas the corresponding CDF is given by (25), at the top of the next page. Notably, the involved integral can be expressed in closed-form with the aid of [38, eq. (8.350.1)]. As a result, by performing the necessary change of variables and substituting in (25), the following expression is deduced

$$
\begin{gathered}
F_{\gamma}(\gamma)=\mathcal{A} \sum_{l=0}^{m_{1}-m_{2}-\frac{1}{2}} \frac{\sqrt{\pi} \Gamma\left(m_{1}-m_{2}+l+\frac{1}{2}\right)}{l ! 2^{l-\frac{1}{2}} \mathcal{B}^{m_{1}+m_{2}} \Gamma\left(m_{1}-m_{2}-l+\frac{1}{2}\right)} \\
\times \gamma\left(m_{1}-m_{2}+l+\frac{1}{2}, \mathcal{B} \sqrt{\gamma}\right)
\end{gathered}
$$

which is also valid for the case that $m_{1}-m_{2} \pm \frac{1}{2} \in \mathbb{N}$. 


$$
F_{\gamma}(\gamma)=\sum_{l=0}^{m_{1}-m_{2}-\frac{1}{2}} \frac{\mathcal{A} \sqrt{\pi} \Gamma\left(m_{1}-m_{2}+l+\frac{1}{2}\right)}{l ! \Gamma\left(m_{1}-m_{2}-l+\frac{1}{2}\right)(2 \mathcal{B})^{l+\frac{1}{2}}} \int_{0}^{\gamma} x^{\frac{m_{1}+m_{2}-l}{2}-\frac{5}{4}} e^{-\mathcal{B} x} \mathrm{~d} x
$$

\section{B. V2V systems impaired by IQI}

1) TX impaired by IQI: Using (12), in the case of singlecarrier V2V communication with only TX impaired with IQI, the corresponding OP can be expressed as

$$
P_{\text {out }}=F_{\gamma}\left(\frac{1}{\left|K_{1}^{t}\right|^{2}\left(\frac{1}{\gamma_{t h}}-\frac{1}{I R R_{t}}\right)}\right)
$$

with $\gamma_{t h} \leq I R R_{t}$. By also using (23) and (25), eq. (27) can be expressed by (28), top of the next page, which for the special case that $m_{1}-m_{2} \pm \frac{1}{2} \in \mathbb{N}$ can be further simplified to

$$
\begin{aligned}
P_{\text {out }}= & \sum_{l=0}^{m_{1}-m_{2}-\frac{1}{2}} \frac{2^{\frac{1}{2}-l} \Gamma\left(m_{1}-m_{2}+l+\frac{1}{2}\right)}{l ! \mathcal{B}^{m_{1}+m_{2}} \Gamma\left(m_{1}-m_{2}-l+\frac{1}{2}\right)} \\
& \quad \times \gamma\left(m_{1}-m_{2}+l+\frac{1}{2}, \frac{\mathcal{B}}{\left|K_{1}^{t}\right| \sqrt{\frac{1}{\gamma_{t h}}-\frac{1}{I R R_{t}}}}\right)
\end{aligned}
$$

where $\gamma(a, x)$ is the lower incomplete gamma function [37].

2) $R X$ impaired by IQI: With the aid of (15), the corresponding OP in this scenario is given by

$$
P_{\text {out }}=F_{\gamma_{\text {ideal }}}\left(\frac{1+\frac{1}{I R R_{r}}}{\frac{1}{\gamma_{t h}}-\frac{1}{I R R_{r}}}\right)
$$

with $\gamma_{t h} \leq I R R_{r}$. Using (23) and (25), it follows that

$$
\begin{aligned}
& P_{\text {out }}=\frac{\mathcal{A} \Gamma\left(m_{2}-m_{1}\right)}{m_{1} 2^{m_{1}-m_{2} \mathcal{B}^{m_{2}-m_{1}}}}\left(\frac{1+\frac{1}{\operatorname{IRR} R_{r}}}{\frac{1}{\gamma_{\text {th }}}-\frac{1}{\operatorname{IRR_{r}}}}\right)^{m_{1}} \\
& \times{ }_{1} F_{2}\left(m_{1} ; m_{1}+1, m_{1}-m_{2}+1 ; \frac{\mathcal{B}^{2}}{4} \frac{1+\frac{1}{I R R_{r}}}{\frac{1}{\gamma_{t h}}-\frac{1}{I R R_{r}}}\right) \\
& +\frac{\mathcal{A} \Gamma\left(m_{1}-m_{2}\right)}{m_{2} 2^{1-m_{1}+m_{2} \mathcal{B}^{m_{2}-m_{1}}}}\left(\frac{1+\frac{1}{\operatorname{IRR_{r}}}}{\frac{1}{\gamma_{t h}}-\frac{1}{I R R_{r}}}\right) \\
& \times{ }_{1} F_{2}\left(m_{2} ; m_{2}+1, m_{2}-m_{1}+1 ; \frac{\mathcal{B}^{2}}{4} \frac{1+\frac{1}{I R R_{r}}}{\frac{1}{\gamma_{t h}}-\frac{1}{I R R_{r}}}\right)
\end{aligned}
$$

which for $m_{1}-m_{2} \pm \frac{1}{2} \in \mathbb{N}$ can be expressed as

$$
\begin{aligned}
P_{\text {out }}=\sum_{l=0}^{m_{1}-m_{2}-\frac{1}{2}} \frac{\mathcal{A} \sqrt{\pi} \Gamma\left(m_{1}-m_{2}+l+\frac{1}{2}\right)}{l ! 2^{l-\frac{1}{2}} \mathcal{B}^{m_{1}+m_{2}} \Gamma\left(m_{1}-m_{2}-l+\frac{1}{2}\right)} \\
\quad \times \gamma\left(m_{1}-m_{2}+l+\frac{1}{2}, \mathcal{B} \sqrt{\frac{1+\frac{1}{I R R_{r}}}{\frac{1}{\gamma_{t h}}-\frac{1}{I R R_{r}}}}\right) .
\end{aligned}
$$

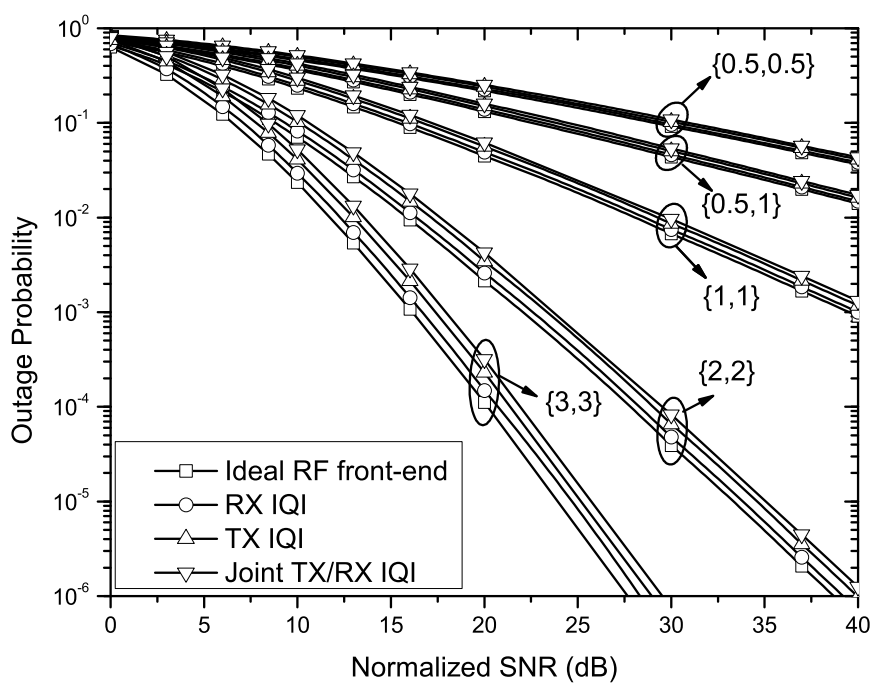

Fig. 2. OP versus normalized outage SNR, when $I R R=20 \mathrm{~dB}$ and $\phi=3^{\circ}$.

3) Joint TX/RX impaired by IQI: With the aid of (20), the corresponding $\mathrm{OP}$ in the case is expressed as follows

$$
P_{\text {out }}=F_{\gamma_{\text {ideal }}}\left(\frac{\left|K_{1}^{r}\right|^{2}+\left|K_{2}^{r}\right|^{2}}{\frac{\left|\xi_{11}\right|^{2}}{\gamma_{t h}}-\left(\left|\xi_{12}\right|^{2}+\left|\xi_{21}\right|^{2}\right)}\right)
$$

where $\gamma_{\mathrm{th}} \leq\left|\xi_{11}\right|^{2} /\left(\left|\xi_{12}\right|^{2}+\left|\xi_{21}\right|^{2}\right)$. It is evident that by following the same methodology, equation (33) can be expressed in closed-form according to (III-B3), top of the next page, which for the specific case that $m_{1}-m_{2} \pm \frac{1}{2} \in \mathbb{N}$, it can be further simplified to the following expression

$$
\begin{aligned}
P_{\text {out }} & =\frac{\mathcal{A} \sqrt{\pi}}{\mathcal{B}^{m_{1}+m_{2}}} \sum_{l=0}^{m_{1}-m_{2}-\frac{1}{2}} \frac{\Gamma\left(m_{1}-m_{2}+l+\frac{1}{2}\right)}{l ! 2^{l-\frac{1}{2}} \Gamma\left(m_{1}-m_{2}-l+\frac{1}{2}\right)} \\
& \times \gamma\left(m_{1}-m_{2}+l+\frac{1}{2}, \mathcal{B} \sqrt{\frac{\left|K_{1}^{r}\right|^{2}+\left|K_{2}^{r}\right|^{2}}{\frac{\left|\xi_{11}\right|^{2}}{\gamma_{t h}}-\left(\left|\xi_{12}\right|^{2}+\left|\xi_{21}\right|^{2}\right)}}\right) .
\end{aligned}
$$

It is noted here that when the distance separating the involved vehicles is larger than $5 \mathrm{~m}$, the corresponding line of sight (LOS) component tends to disappear resulting to more severe fading conditions [14]. This also holds for double Rayleigh fading scenarios [39]-[41], which constitutes a specific case of the double Nakagami $-m$ for $m_{2}=m_{1}=1$.

\section{NumericAl Results}

In this section, we analyze the effects of IQI on the performance of wireless $\mathrm{V} 2 \mathrm{~V}$ communications over double Nakagami- $m$ fading channels in terms of the corresponding 


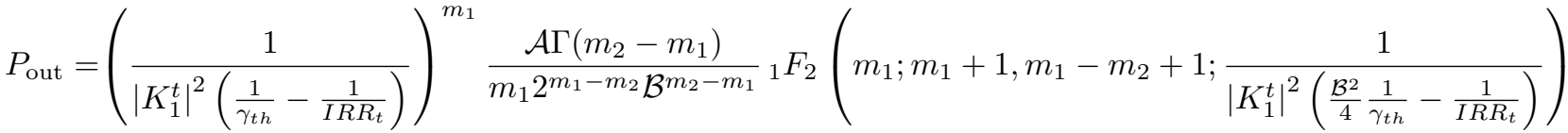

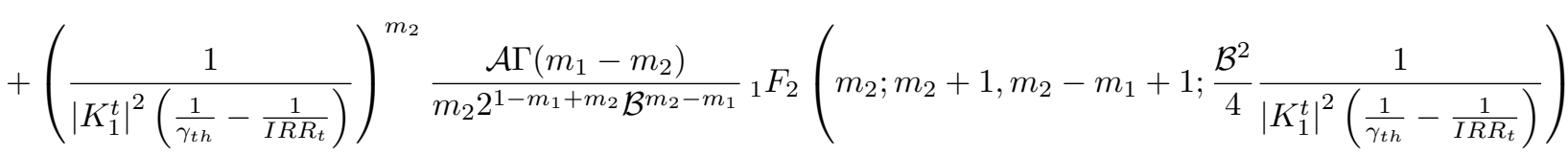

$$
\begin{aligned}
& P_{\text {out }}=\frac{\mathcal{A} \Gamma\left(m_{2}-m_{1}\right)}{m_{1} 2^{m_{1}-m_{2} \mathcal{B}^{m_{2}-m_{1}}}}\left(\frac{\left|K_{1}^{r}\right|^{2}+\left|K_{2}^{r}\right|^{2}}{\frac{\left|\xi_{11}\right|^{2}}{\gamma_{\text {th }}}-\left(\left|\xi_{12}\right|^{2}+\left|\xi_{21}\right|^{2}\right)}\right)^{m_{1}}{ }_{1} F_{2}\left(m_{1} ; m_{1}+1, m_{1}-m_{2}+1 ; \frac{\mathcal{B}^{2}}{4} \frac{\left|K_{1}^{r}\right|^{2}+\left|K_{2}^{r}\right|^{2}}{\frac{\left|\xi_{11}\right|^{2}}{\gamma_{t h}}-\left(\left|\xi_{12}\right|^{2}+\left|\xi_{21}\right|^{2}\right)}\right) \\
& +\frac{\mathcal{A} \Gamma\left(m_{1}-m_{2}\right)}{m_{2} 2^{1-m_{1}+m_{2}} \mathcal{B}^{m_{2}-m_{1}}}\left(\frac{\left|K_{1}^{r}\right|^{2}+\left|K_{2}^{r}\right|^{2}}{\frac{\left|\xi_{11}\right|^{2}}{\gamma_{t h}}-\left(\left|\xi_{12}\right|^{2}+\left|\xi_{21}\right|^{2}\right)}\right)^{m_{2}}{ }_{1} F_{2}\left(m_{2} ; m_{2}+1, m_{2}-m_{1}+1 ; \frac{\mathcal{B}^{2}}{4} \frac{\left|K_{1}^{r}\right|^{2}+\left|K_{2}^{r}\right|^{2}}{\frac{\left.\xi_{11}\right|^{2}}{\gamma_{t h}}-\left(\left|\xi_{12}\right|^{2}+\left|\xi_{21}\right|^{2}\right)}\right) .
\end{aligned}
$$

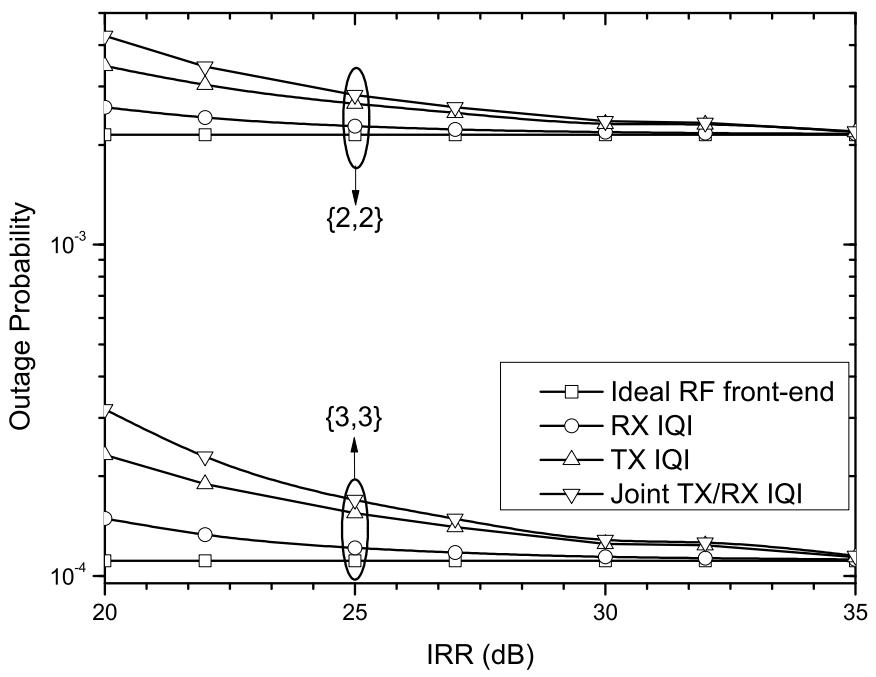

Fig. 3. OP as a function of the IRR, when $\mathrm{SNR}=20 \mathrm{~dB}$.

OP. The notation $m=\left\{m_{1}, m_{2}\right\}$ accounts for up to double Nakagami- $m$ channels with fading severity factors $m_{1}$, and $m_{2}$. Furthermore, we assume that SNR is normalized with respect to $\gamma_{\text {th }}$, which implies that the OP is evaluated as a function of $\gamma / \gamma_{\text {th }}$. To this end, Fig. 2 illustrates the OP versus the normalized SNR for the different considered TX/RX scenarios. Specifically, we compare the OP between the ideal $\mathrm{RF}$ front-end, the RX imbalanced, the TX imbalanced and the joint TX/RX imbalanced cases when the $I R R=20 \mathrm{~dB}$ and $\phi=3^{\circ}$. Furthermore, we consider the following severity of fading cases, $m=\{0.5,0.5\}, m=\{0.5,1\}, m=\{1,1\}$, $m=\{2,2\}$ and $m=\{3,3\}$. It is shown that the performance degradation created by the IQI is rather limited compared to the detrimental effects of the double Nakagami- $m$ fading. For example, the OP value in the case of double Rayleigh fading, i.e. $m=\{1,1\}$, for $\gamma / \gamma_{t h}=10 \mathrm{~dB}$ is nearly $50 \%$ less that the OP in the case of double Nakagami- $m$ fading, with $m=\{0.5,0.5\}$. In addition, in the case of double Rayleigh fading conditions, the assumption of ideal RF front-end results to around $20 \%$ error in the corresponding OP. These results highlight the importance of both accurate channel characterization and precise consideration of RF impairments, in the realistic performance analysis and design of wireless communication systems. It is also interesting to note that the effects of TX IQI only on the OP degradation are more severe than the corresponding effects of RX IQI only case. The underlying reason for this observation is that the SINR is higher in the case of RX IQI only than in the case of TX IQI only, since the noise is multiplied by $\left(\left|K_{1}^{r}\right|^{2}+\left|K_{2}^{r}\right|^{2}\right)$, which for practical values of IQI levels does not exceed 1.

Fig. 3 illustrates the effects of the IRR on the OP for the different considered TX/RX scenarios assuming double Nakagami- $m$ fading channels with $m=\{2,2\}$ and $m=$ $\{3,3\}$. It is evident that the OP is lower in the latter scenario while it improves as IRR increases. For example, when $m=\{3,3\}$ with $\gamma / \gamma_{t h}=20 \mathrm{~dB}$ and $I R R=25 \mathrm{~dB}$ exhibits a $47 \%$ OP reduction compared to the $I R R=20 \mathrm{~dB}$ case, for of joint TX/RX IQI effects. By also recalling that the IRR of practical RF front-ends lies in the range of $20-40 \mathrm{~dB}$, it becomes evident that accounting for RF impairments is crucial.

\section{CONCLUSIONS}

This paper investigated the OP performance of $\mathrm{V} 2 \mathrm{~V}$ communication systems over double Nakagami- $m$ channels in the presence of IQI at the RF front-end. We considered three scenarios in our analysis corresponding to ideal TX with I/Q imbalanced RX, I/Q imbalanced TX with ideal RX, and joint I/Q imbalanced TX and RX. The ideal case was also taken into consideration for comparison and the derived analytic results were validated through extensive comparisons with computer simulations. It was shown that the severity of fading affects significantly the corresponding performance and that assuming ideal RF front-end results to around $20 \%$ erroneous OP. This 
highlights the importance of accurate channel characterization and consideration for RF impairments in the analysis, design and deployment of $\mathrm{V} 2 \mathrm{~V}$ communication systems.

\section{ACKNOWLEDGMENTS}

This work was supported by the Finnish Funding Agency for Technology and Innovation (Tekes) under the project entitled "Energy-Efficient Wireless Networks and Connectivity of Devices-Systems (EWINE-S)", and by the Academy of Finland under the projects No. 251138 Digitally-Enhanced RF for Cognitive Radio Devices and No. 284694 "Fundamentals of Ultra Dense 5G Networks with Application to Machine Type Communication".

\section{REFERENCES}

[1] M. Kihl, K. Bur, F. Tufvesson, and J. Aparicio Ojea, "Simulation modelling and analysis of a realistic radio channel model for V2V communications," in Int. Congr. on Ultra Modern Telecommunications and Control Systems and Workshops, Moscow, Oct 2010, pp. 981-988.

[2] D. Zogas, G. K. Karagiannidis and S Kotsopoulos, "On the average output SNR in selection combining with three correlated branches over Nakagami $-m$ fading channels," IEEE Trans. Wireless Commun., vol. 3 , no. 1 , pp. 25-28, Jan. 2004

[3] G. K Karagiannidis, D. Zogas, N.C Sagias, T. A. Tsiftsis, P. T. Mathiopoulos, "Multihop communications with fixed-gain relays over generalized fading channels," IEEE GLOBECOM '04, pp. 36-40.

[4] P. C. Sofotasios and S. Freear, "The $\kappa-\mu /$ gamma Composite Fading Model," IEEE ICWITS '10, Honolulu, HI, USA, Aug. 2010.

[5] D. Zogas and G. K. Karagiannidis, "Infinite-series representations associated with the bivariate Rician distribution and their applications," IEEE Trans. Commun., vol. 53, no. 11, pp. 1790-1794, Nov. 2005.

[6] P. C. Sofotasios and S. Freear, "The $\alpha-\kappa-\mu$ Extreme Distribution: Characterizing Non Linear Severe Fading Conditions," ATNAC '11, Melbourne, Australia, Nov. 2011.

[7] D.S. Michalopoulos, G.K. Karagiannidis, T. A. Tsiftsis, and R. K. Mallik, "An optimized user selection method for cooperative diversity systems," IEEE GLOBECOM '06, pp. 1-6.

[8] P. C. Sofotasios, T. A. Tsiftsis, M. Ghogho, L. R. Wilhelmsson and M.Valkama, "The $\eta-\mu /$ Inverse-Gaussian Distribution: A Novel Physical Multipath /Shadowing Fading Model," IEEE ICC '13, Budapest, Hungary, June 2013.

[9] Z Hadzi-Velkov, N. Zlatanov, G.K. Karagiannidis "On the second order statistics of the multihop Rayleigh fading channel," IEEE Trans. Commun., vol. 57, no. 6, pp. 1815-1823, June 2009.

[10] P. C. Sofotasios, T. A. Tsiftsis, K. Ho-Van, S. Freear, L. R. Wilhelmsson, and M. Valkama, "The $\kappa-\mu /$ Inverse-Gaussian Composite Statistical Distribution in RF and FSO Wireless Channels," in IEEE VTC '13Fall, Las Vegas, USA, pp. 1-5, Sep. 2013.

[11] D. S Michalopoulos, A. S. Lioumpas, G. K. Karagiannidis and R. Schober "Selective cooperative relaying over time-varying channels," IEEE Trans. Commun., vol. 58, no. 8, pp. 2402-2412, Aug. 2010.

[12] P. C. Sofotasios, M. Valkama, Yu. A. Brychkov, T. A. Tsiftsis, S. Freear and G. K. Karagiannidis, "Analytic Solutions to a Marcum $Q$-FunctionBased Integral and Application in Energy Detection," in CROWNCOM 14, Oulu, Finland, pp. 260-265, June 2014.

[13] P. C. Sofotasios, T. A. Tsiftsis, Yu. A. Brychkov, S. Freear, M. Valkama, and G. K. Karagiannidis, "Analytic Expressions and Bounds for Special Functions and Applications in Communication Theory," IEEE Trans. Inf. Theory, vol. 60, no. 12, pp. 7798-7823, Dec. 2014.

[14] A. Molisch, F. Tufvesson, J. Karedal, and C. Mecklenbrauker, "A survey on vehicle-to-vehicle propagation channels," IEEE Wireless Commun. Mag., vol. 16, no. 6, pp. 12-22, Dec. 2009.

[15] H. Ilhan, M. Uysal, and I. Altunbas, "Cooperative diversity for intervehicular communication: Performance analysis and optimization," IEEE Trans. Veh. Commun., vol. 58, no. 7, pp. 3301-3310, Sep. 2009.

[16] I. Sen, D. Matolak, "Vehicle-Vehicle channel models for the $5 \mathrm{GHz}$ band," IEEE Trans. Intell. Transp. Syst., vol. 9, no. 2, pp. 235-245, Jun. 2008.
[17] M. Mokhtar, A.-A. A. Boulogeorgos, G. K. Karagiannidis, and N. AlDhahir, "OFDM Opportunistic Relaying Under Joint Transmit/Receive I/Q Imbalance,” IEEE Trans. Commun., vol. 62, no. 5, pp. 1458-1468, May 2014.

[18] E. Bjornson, M. Matthaiou, and M. Debbah, "A new look at dual-hop relaying: Performance limits with hardware impairments," IEEE Trans. Commun., vol. 61, no. 11, pp. 4512-4525, November 2013.

[19] T. Schenk, RF Imperfections in High-Rate Wireless Systems. The Netherlands: Springer, 2008.

[20] A.-A. A. Boulogeorgos, N. Chatzidiamantis, G. K. Karagiannidis, and L. Georgiadis, "Energy detection under RF impairments for cognitive radio," in Proc. IEEE ICC 15, London, United Kingdom, June 2015.

[21] J. Qi and S. Aissa, "Analysis and compensation of I/Q imbalance in MIMO transmit-receive diversity systems," IEEE Trans. Commun., vol. 58 , no. 5, pp. 1546-1556, May 2010.

[22] Y. Zou, M. Valkama, and M. Renfors, "Digital compensation of $\mathrm{I} / \mathrm{Q}$ imbalance effects in space-time coded transmit diversity systems," IEEE Trans. Signal Process., vol. 56, no. 6, pp. 2496-2508, JUN 2008.

[23] J. Qi, S. Aissa, and M.-S. Alouini, "Dual-hop amplify-and-forward cooperative relaying in the presence of Tx and Rx in-phase and quadraturephase imbalance," IET Commun., vol. 8, no. 3, pp. 287-298, Feb 2014.

[24] A. Gokceoglu, Y. Zou, M. Valkama, P. C. Sofotasios, P. Mathecken, D. Cabric, "Mutual Information Analysis of OFDM Radio Link under Phase Noise, IQ Imbalance and Frequency-Selective Fading Channel," IEEE Trans. Wireless Commun., vol.12, no.6, pp. 3048-3059, June 2013.

[25] J. Li, M. Matthaiou, and T. Svensson, "I/Q imbalance in AF dual-hop relaying: Performance analysis in Nakagami- $m$ fading," IEEE Trans. Commun., vol. 62, no. 3, pp. 836-847, Mar. 2014

[26] A. Gokceoglu, Y. Zhou, M. Valkama, and P. C. Sofotasios, "MultiChannel Energy Detection under Phase Noise: Analysis and Mitigation," ACM/Springer Journal on Mobile Networks and Applications (MONET), vol. 19 , no. 4, pp. 473-486, Aug. 2014

[27] C. Studer, M. Wenk, and A. Burg, "System-level implications of residual transmit-RF impairments in MIMO systems," in Proc. of the 5th European Conf. on Ant.s and Prop., April 2011, pp. 2686-2689.

[28] A. Gokceoglu, S. Dikmese, M. Valkama, and M. Renfors, "Energy Detection under IQ Imbalance with Single- and Multi-Channel DirectConversion Receiver: Analysis and Mitigation," IEEE J. Sel. Areas Commun., vol. 32, no. 3, pp. 411-424, March 2014

[29] G. K. Karagiannidis, N. C. Sagias, and P. T. Mathiopoulos, "N*Nakagami: A novel stochastic model for cascaded fading channels," IEEE Trans. on Commun., vol. 55, no. 8, pp. 1453-1458, Aug 2007.

[30] B. Razavi, RF microelectronics. Prentice Hall New Jersey, 1998, vol. 1.

[31] S. Mirabbasi and K. Martin, "Classical and modern receiver architectures," IEEE Commun. Mag., vol. 38, no. 11, pp. 132-139, Nov 2000.

[32] L. Anttila, M. Valkama, and M. Renfors, "Circularity-based I/Q imbalance compensation in wideband direct-conversion receivers," IEEE Trans. Veh. Commun., vol. 57, no. 4, pp. 2099-2113, July 2008.

[33] M. Valkama, J. Pirskanen, and M. Renfors, "Signal processing challenges for applying software radio principles in future wireless terminals: an overview," Int. J. Commun. Syst., vol. 15, no. 8, pp. 741-769, 2002.

[34] P. Rykaczewski, M. Valkama, and M. Renfors, "On the connection of I/Q imbalance and channel equalization in direct-conversion transceivers," IEEE Trans. Veh. Technol., vol. 57, no. 3, pp. 1630-1636, May 2008.

[35] S. J. Grant and J. K. Cavers, "Analytical calculation of outage probability for a general cellular mobile radio system," in IEEE Vehicular Technology Conf., vol. 3. Amsterdam: IEEE, 1999, pp. 1372-1376.

[36] M. K. Simon and M.-S. Alouini, Digital communication over fading channels. John Wiley \& Sons, 2005, vol. 95.

[37] A. P. Prudnikov, Y. A. Brychkov, and O. I. Marichev, Inegrals and Series, Vol. 2: Special Functions. Gordon and Breach Science Publishers, 1992.

[38] I. S. Gradshteyn and I. M. Ryzhik, Table of Integrals, Series, and Products, 6th ed. New York: Academic, 2000.

[39] V. Erceg, S. Fortune, J. Ling, A. Rustako, and R. Valenzuela, "Comparisons of a computer-based propagation prediction tool with experimental data collected in urban microcellular environments," IEEE J. Sel. Areas Commun., vol. 15, no. 4, pp. 677-684, May 1997.

[40] H. Hadizadeh, S. Muhaidat, and I. Bajic, "Impact of imperfect channel estimation on the performance of inter-vehicular cooperative networks," in IEEE Biennial Symp. on Comm., Kingston, May 2010, pp. 373-376.

[41] M. Shirkhani, Z. Tirkan, and A. Taherpour, "Performance analysis and optimization of two-way cooperative communications in inter-vehicular networks," in IEEE Int. Conf. on Wireless Communications Signal Processing, Huangshan, Oct 2012, pp. 1-6. 